(c) Elsevier/INRA

Article original

\title{
Schémas de sélection : de la représentation généalogique au modèle statistique. Élaboration du modèle
}

\author{
B Mangin ${ }^{1}, \mathrm{P}_{\text {Vincourt }}{ }^{2}$ \\ ${ }^{1}$ Institut national de la recherche agronomique, station de biométrie \\ et d'intelligence artificielle de Toulouse, Chemin de Bordes Rouge, Auzeville, BP 27, \\ 31326 Castanet-Tolosan Cedex; \\ ${ }^{2}$ Rustica Semences, domaine Sandreau, Daux, 31700 Blagnac, France
}

(Reçu le 7 mars 1990; accepté le 4 décembre 1991)

\begin{abstract}
Résumé - Nous présentons une méthode s'adressant plus particulièrement à des sélectionneurs d'espèces végétales qui veulent modéliser leurs observations dans le cadre des modèles linéaires multivariables à effets aléatoires et fixes. Cette méthode est basée sur une description par un schéma de la généalogie des plantes manipulées dans un protocole de sélection. Nous proposons des règles de construction du schéma et les illustrons sur un exemple. En utilisant une représentation de ce schéma à l'aide d'un graphe orienté, nous donnons une méthode pour construire le modèle statistique pour la valeur génétique des individus et énonçons les règles qui permettent de trouver les variance-covariances entre apparentés.
\end{abstract}

sélection végétale / modèle statistique / modèle linéaire / graphe

Summary - Selection schemes : from the genealogical representation to the statistical model. Model development. We present a methodology addressed particularly to plant breeders who want to model their data by a linear multivariate mixed model. The first part of this methodology is the description of the genealogy for the plants used in the protocol of selection, by a diagram. We propose the rules for drawing the diagram and give an example. Using an oriented graph related to the diagram we present the second part of the methodology for constructing the statistical model for the individual genetic value and set forth a rule for finding the variance-covariances between related individuals.

plant breeding / statistical model / linear model / graph 


\section{INTRODUCTION}

Pour prédire la valeur génétique d'un individu pour un caractère quantitatif dans le cadre des protocoles de sélection utilisés en amélioration des plantes, il est maintenant classique d'essayer d'utiliser au maximum toute l'information disponible, c'est-à-dire toutes les mesures faites sur l'individu et sur ses apparentés (Gallais, 1990). Nous nous placerons dans le cas où un modèle linéaire à effets aléatoires ou fixes permet de modéliser les observations et nous considérerons que la méthode statistique permettant la prédiction de la valeur génétique est le BLUP (Henderson, 1973). La seule chose requise est alors de pouvoir écrire l'espérance et la matrice de variance-covariance des observations qui constitueront l'information. Pour ce faire, nous supposerons que l'observation est la somme de 2 variables aléatoires. La première d'espérance nulle est la valeur génétique de l'individu (pour un lieu, une année et un plan d'expérience donnés). La seconde est liée au milieu, son espérance et sa variance sont modélisées en tenant compte du plan d'expérience mis en place dans le milieu. Nous allons proposer une méthode pour trouver la matrice de variance-covariance des valeurs génétiques en les modélisant par un modèle statistique qui ne dépendra que de la généalogie des individus. Ce modèle sera trouvé à partir d'un schéma qui décrira le protocole de croisement ayant permis d'obtenir les individus observés en plein champ. La méthode de construction du modèle est basée sur des graphes orientés.

Les graphes sont des objets qui ont été déjà largement utilisés en génétique des populations et dans les analyses de pédigrés. Leur utilisation, soit pour obtenir les coefficients d'identité par descendance (Maruyama et Yasuda, 1970), soit pour trouver la loi conjointe des observations (Cannings et al, 1978) est toujours subordonnée à un modèle de transmission des gènes, ce qui n'est pas notre cas. D'autre part, les graphes qui vont vous permettre de trouver un modèle statistique et ceux qui sont utilisés dans les analyses de pédigrés n'ont pas du tout le même niveau de complexité. La différence réside dans le fait que pour les premiers, un sommet du graphe est un type de géniteur (ou d'ancêtre) tandis que pour les seconds, chaque sommet est un individu.

Nous allons d'abord clairement exposer ce que nous entendons par descendance, type de descendance et type de géniteur. De plus, pour chaque descendance, nous rappellerons quels sont les ancêtres qui sont intéressants pour la généalogie. Puis nous poserons les bases de construction du schéma représentatif d'un protocole de création de descendances. De ce schéma, nous déduirons le modèle statistique pour la valeur génétique de chaque type de descendance, en utilisant des graphes orientés. Nous terminerons en donnant des règles algébriques permettant de construire simplement la matrice de variance-covariance recherchée.

\section{DESCENDANCES, TYPE DE DESCENDANCE ET TYPE DE GÉNITEUR}

En amélioration des plantes, on parle de descendance lorsque l'on a des individus qui ont tous été obtenus : 
- soit à partir d'une plante par autofécondation ou clonage; pour la descendance d'autofécondation ou de clones obtenue, le seul ancêtre retenu dans sa généalogie est la plante autofécondée ou clonée;

- soit à partir d'une plante servant de mère et d'un pollen non contrôlé; pour la descendance de demi-frères ou sœurs obtenue; seule la mère est présente dans la généalogie;

- soit à partir d'une plante et d'un testeur (ensemble de plantes à base plus ou moins étroite, voire réduite à génotype); pour la descendance en croisement avec le testeur, le seul ancêtre présent dans sa généalogie est la plante croisée avec le testeur;

- soit à partir d'une plante mère et d'une plante père par pollinisation contrôlée ; c'est le seul type de croisement où les 2 parents entrent dans la généalogie de la descendance de plein-frères ou sœurs obtenue.

À cette notion de descendance vient se rajouter une notion plus floue de population qui sont des plantes dont la généalogie est oubliée, qui sont regroupées par le sélectionneur et forment le matériel de départ du protocole de sélection.

Un type de descendance sera le regroupement de toutes les descendances qui possèdent une généalogie "comparable», et un type de géniteur sera l'ensemble des individus qui sont les ancêtres «comparables» dans la généalogie d'un type de descendance. Cette notion de «comparable» aurait pu être explicitée en étudiant les graphes généalogiques de chaque plante prise individuellement; nous avons préféré présenter une démarche plus simple qui consiste à regrouper automatiquement les plantes dont les généalogies sont «comparables». Nous verrons, dans le paragraphe suivant, que cette démarche qui conduit à un schéma simple, peut être comprise de façon intuitive.

\section{Décrire la généalogie des plantes à l'aide d'un schéma}

Nous allons pour illustrer notre propos, utiliser le protocole de sélection proposé par Gallais (1977). Ce protocole est basé sur 2 populations de départ A et B, pour lesquelles les descendances de A sont obtenues par autofécondation et pollinisation non contrôlée avec le pollen de la population $B$. Une étape de test, puis de sélection, permet de choisir les individus des populations de départ qui serviront à créer les plantes de deuxième génération obtenues par pollinisation contrôlée entre 2 plantes de la $S_{1}$ (ensemble des descendances obtenues par autofécondation de plantes d'une population de départ). Nous ne nous intéresserons pas à l'étape de test et de sélection, notre propos étant de décrire la généalogie des plantes impliquées dans le protocole proposé. Comme il est fastidieux de vouloir représenter la généalogie de toutes les plantes prises individuellement, notre idée est de poser des règles pour dessiner un schéma dans lequel bien qu'aucune plante ne sera explicitement pointée, toutes les plantes trouveront leur place.

\section{Règles de construction d'un schéma}

Nous allons représenter par un cercle les populations de départ et par un carré les populations de testeurs. Puis nous représenterons par un unique point intérieur au cercle tous les individus ayant servi de la même façon pour créer des descendances, 
ce que nous appellons un type de géniteur. À partir de ces points, nous dessinerons la manipulation effectuée comme proposé par la figure 1.

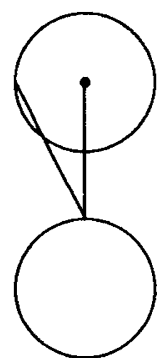

(1)

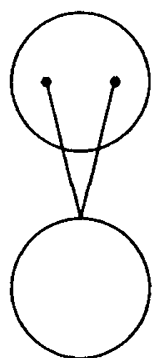

(2)

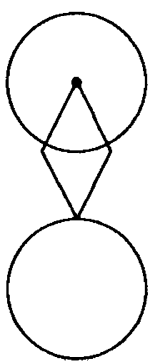

(3)

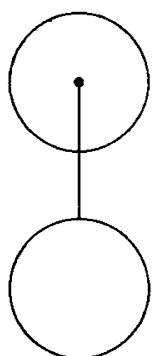

(4)

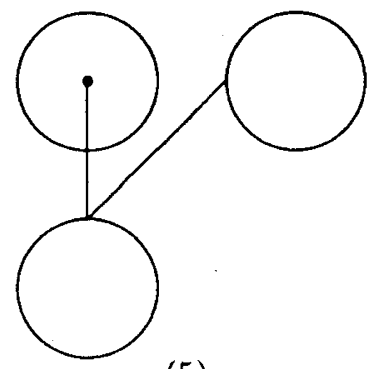

(5)

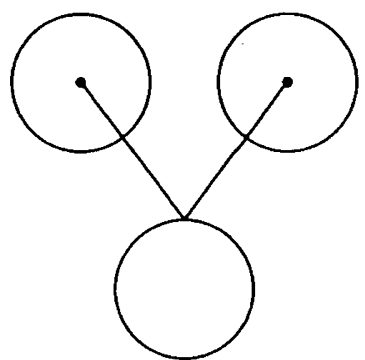

(6)

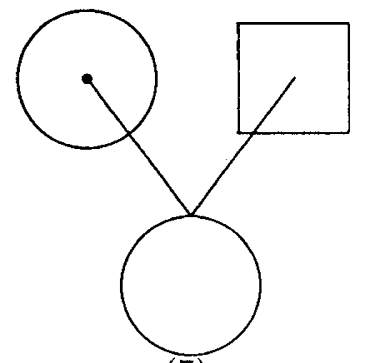

(7)

Fig 1. Manipulations génétiques. (1) Création de descendances de demi-frères intrapopulation; (2) Création de descendances de plein-frères intra-population; (3) Création de descendances obtenues par autofécondation; (4) Création de descendances obtenues par clonage; (5) Création de descendances de demi-frères inter-populations; (6) Création de descendances de plein-frères inter-populations; ( 7 ) Création de descendances obtenues par croisement avec des testeurs.

Nous disposons alors sur le schéma, de cercles représentant maintenant chacun un type de descendance. Nous avons choisi volontairement de ne pas chercher à représenter l'information (car elle n'est pas nécessaire pour trouver le modèle) qui au niveau des plantes individuelles des populations de départ permettait de savoir :

- si une plante n'avait jamais servi;

- si la même plante avait servi à créer plus d'un type de descendances.

La figure 2 présente le schéma pour les premières manipulations des plantes de la population A dans le protocole de Gallais (1977).

Intéressons-nous maintenant à la suite du protocole. Étudions, en particulier, les pollinisations contrôlées à l'intérieur de la $S_{1}$ (cercle $c_{3}$ de la figure 1). Cette pollinisation dans la $S_{1}$ va permettre, soit de recombiner la même plante de $A$, ce qui sera fait en prenant un père et une mère dans la même descendance d'autofécondation; soit de recombiner 2 plantes différentes de A, ce qui sera fait en prenant un père et une mère dans 2 descendances différentes. Du point de vue de la généalogie pour les descendants du croisement père $\times$ mère envisagé, il est très différent d'avoir un seul ancêtre dans $\mathrm{A}$ ou d'en avoir deux. Or, à cette étape, 


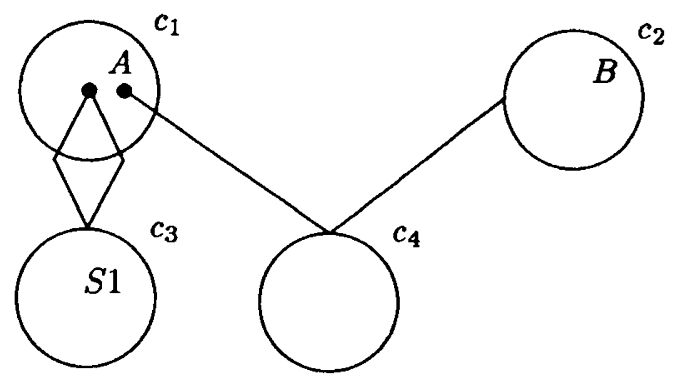

Fig 2. Schéma représentant la première génération du protocole de Gallais (1977). $c_{1}$ représente toute la population $A ; c_{2}$ représente toute la population $B ; c_{3}$ représente l'ensemble de toutes les descendances obtenues par autofécondation à partir d'une plante de $A ; c_{4}$ représente l'ensemble de toutes les descendances obtenues par pollinisation non contrôlée à partir d'une plante de $A$.

nous ne disposons que d'un cercle dans le schéma pour représenter la $S_{1}$. Nous avons dû faire des choix pour pouvoir dessiner à partir de cet unique cercle, 2 types différents de descendances. Ces choix, que nous avons voulu généraux, nous ont amenés à définir des règles concernant les cercles et les points intérieurs aux cercles du schéma.

- Un cercle du schéma représente soit une population de départ, soit un type de descendance.

- Un point à l'intérieur d'un cercle représente un type de géniteur c'est-à-dire l'ensemble des plantes ayant servi de la même façon pour créer une descendance (une même plante peut donc être représentée par plus d'un point).

- Lorsqu'un cercle a plus d'un point ancêtre dans un cercle antérieur représentant un type de descendance, ces points représentent, soit la même plante, soit des plantes qui ont les mêmes parents mais jamais des plantes qui appartiennent à des descendances différentes.

- Lorsqu'un cercle a plus d'un point ancêtre dans un cercle antérieur, on conviendra de rajouter comme une suite d'égalités entre ces points ancêtres, l'information concernant le fait qu'il s'agit des mêmes plantes.

- Deux cercles du schéma peuvent être équivalents. C'est-à-dire qu'ils représentent les mêmes plantes mais sont nécessaires dans le schéma pour décrire un nouveau cercle.

Pour la même raison que dans le cas des populations, on ne cherche pas à représenter les informations concernant :

- les descendances qui ne servent plus par la suite;

- les descendances qui ne servent qu'à une manipulation si plusieurs manipulations sont représentées dans le cercle. On peut ainsi avoir un schéma pour lequel un cercle sert à 2 manipulations, mais où aucune des descendances ne sert aux 2 manipulations;

- les points qui représentent les mêmes plantes lorsque l'information n'est pas nécessaire.

Le schéma de la figure 3 représente l'ensemble du protocole de Gallais (1977), et permet d'illustrer les choix faits. 


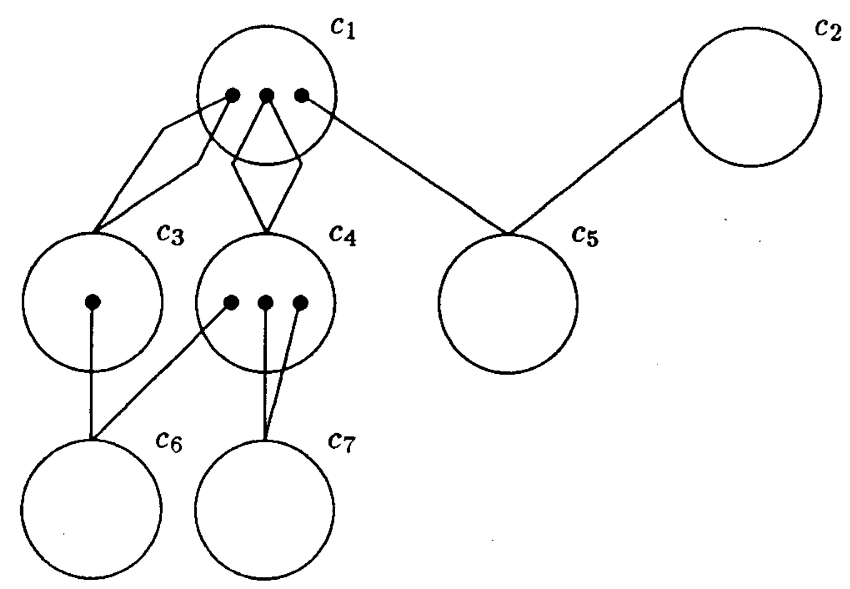

Fig 3. Schéma représentant le protocole de Gallais (1977). $c_{1}$ représente toute la population $A ; c_{2}$ représente toute la population $B ; c_{3}$ et $c_{4}$ sont équivalentes et représentent l'ensemble de toutes les descendances obtenues par autofécondation à partir d'une plante de $A$. Ils sont nécessaires pour décrire la génération suivante; $c_{5}$ représente l'ensemble de toutes les descendances obtenues par pollinisation non contrôlée à partir d'une plante de $A ; c_{6}$ représente l'ensemble de toutes les descendances obtenues à partir d'une pollinisation contrôlée entre 2 descendants d'autofécondation de 2 plantes différentes de $A$; $c_{7}$ représente l'ensemble de toutes les descendances obtenues à partir d'une pollinisation contrôlée entre 2 plantes appartenant à la même descendance d'autofécondation. En effet, les 2 points ancêtres dans $c_{4}$ appartiennent d'après les règles imposées sur les cercles du schéma à la même descendance d'autofécondation.

\section{Graphe orienté d'un cercle du schéma}

Une fois un schéma dessiné, on numérote les $K$ cercles du schéma par $c_{k} ; k=1 \ldots K$ et les $P_{k}$ points intérieurs au cercle $c_{k}$ par $c_{k / p} ; p=1 \ldots P_{k}$. Pour les cercles sans descendance, on crée un point à l'intérieur du cercle, qui représente l'ensemble des individus du cercle (ce point est numéroté logiquement $c_{k / 1}$ ).

On associe de façon bijective à tout cercle du schéma $c_{k}$ le graphe orienté $G^{(k)}$ de la relation «être un descendant par une manipulation élémentaire». Cette relation opère sur l'ensemble des sommets $E^{(k)}$ défini par :

$$
E^{(k)}=\biguplus_{i} E_{i}^{(k)}
$$

où chaque $E_{i}^{(k)}$ est un sous-ensemble de l'ensemble des points intérieur au cercle $c_{i}$. Il est associé au cercle $c_{k}$ considéré et vérifie :

$E_{k}^{(k)}=\left\{c_{k / 1}\right\}$

$E_{i}^{(k)}=0 \quad$ si un individu de $c_{k}$ n'a pas d'ancêtre dans $c_{i}$.

$E_{i}^{(k)}=\left\{\ldots, c_{i / p}, \ldots\right\}$ si $c_{i / p}$ est le point associé à un type de géniteur ancêtre d'un individu de $c_{k}$. Lorsque deux points $c_{i / p}$ et $c_{i / p^{\prime}}$ sont égaux pour $c_{k}$, on élimine le point de plus grand indiçage. 
La relation considérée créant un graphe simple (pas de boucle, au plus un arc entre 2 sommets; Berge, 1983 ; p 5), on convient de noter $G^{(k)}=\left(E^{(k)}, U^{(k)}\right.$, où $U^{(k)}$ est l'ensemble des couples correspondant à un arc du graphe. De plus, on marque le graphe $G^{(k)}$, en conservant dans une table $T^{(k)}$ la nature de la relation ancestrale pour tous les couples de $U^{(k)}$, que l'on désigne de façon abrégée par les lettres $A, P, M, T, C$ ( $A$ pour autofécondation, $P$ pour père, $M$ pour mère, $T$ pour croisement avec un testeur et $C$ pour clone).

Si l'on prend comme exemple, le cercle $c_{6}$ de la figure 2.

$G^{(6)}=\left(E^{(6)}, U^{(6)}\right)$ avec :

$$
\begin{gathered}
E^{(6)}=\left\{c_{1 / 1}, c_{1 / 2}, c_{3 / 1}, c_{4 / 1}, c_{6 / 1}\right\} \\
U^{(6)}=\left\{\left(c_{6 / 1}, c_{4 / 1}\right),\left(c_{6 / 1}, c_{3 / 1}\right),\left(c_{4 / 1}, c_{1 / 2}\right),\left(c_{3 / 1}, c_{1 / 1}\right)\right\}
\end{gathered}
$$

$$
T^{(6)}=\begin{array}{|c|c|}
\left(c_{6 / 1}, c_{4 / 1}\right) & M \\
\left(c_{6 / 1}, c_{3 / 1}\right) & P \\
\left(c_{4 / 1}, c_{1 / 2}\right) & A \\
\left(c_{3 / 1}, c_{1 / 1}\right) & A \\
\hline
\end{array}
$$

\section{Du graphe orienté au modèle}

Les idées directrices de ce paragraphe sont de construire les effets du modèle statistique pour la valeur génétique des individus d'un cercle $c_{k}$ en utilisant le graphe $G^{(k)}$. Notre première idée est d'associer chaque effet du modèle à un sousgraphe de $G^{(k)}$. Cependant, il existe de nombreux sous-graphes dans $G^{(k)}$, aussi nous ne considérons que les sous-graphes qui ont au moins $c_{k / 1}$ comme sommet et dont les sommets ont le même demi-degré intérieur (nombre d'arcs arrivant à un sommet; Berge, $1983 ;$ p 6 ) que dans $G^{(k)}$. Notre deuxième idée est de proposer une «lecture» d'un sous-graphe (noté $G_{l}^{(k)}$ ) qui permette de trouver l'ordre de l'effet associé ainsi que ces niveaux. Pour cela, nous nous intéressons dans chacun des sous-graphes à $S_{l}^{(k)}$ l'ensemble des sommets dont le demi-degré extérieur (nombre d'arcs partant d'un sommet; Berge, 1983; p 6) est nul, c'est-à-dire des sommets n'ayant pas d'ancêtre, ainsi qu'aux chemins liant $c_{k / 1}$ et les sommets de $S_{l}^{(k)}$.

Les propositions 1 et 2 ont pour but de montrer qu'il existe toujours un chemin entre $c_{k / 1}$ et chacun des sommets de $S_{l}^{(k)}$. Ce qui permet de proposer une «lecture» des sous-graphes $G_{l}^{(k)}$ d'où découle le modèle statistique pour la valeur génétique des individus du cercle $c_{k}$. Il reste, bien entendu, à valider un tel modèle. C'est-àdire, à démontrer que cette méthode ne crée ni trop ni trop peu d'effets. C'est l'objet 
de la première partie de l'article de Goffinet et Mangin, 199x (Schéma de sélection : de la représentation généalogique au modèle statistique. Validité asymptotique. Document interne).

\section{Proposition 1}

Un graphe $G^{(k)}$ associé à un cercle du schéma est soit réduit au graphe sans arc d'unique sommet $c_{k / 1}$, soit il admet une racine (point à partir duquel tout sommet peut être atteint; Berge, $1983 ; \mathrm{p} 30)$, qui est $c_{k / 1}$.

\section{Démonstration}

Prenons un graphe $G^{(k)}$ qui n'a qu'un sommet (c'est le cas des graphes associés aux populations de départ des protocoles). Par définition, l'unique sommet de $G^{(k)}$ est $c_{k / 1}$, le graphe étant, de plus, un graphe simple, $G^{(k)}$ n'a pas d'arc.

Prenons alors un graphe $G^{(k)}$ qui a au moins 2 sommets. Soit $c_{i / l}$ un sommet différent de $c_{k / 1}$, il existe donc une suite de manipulations élémentaires qui a permis d'obtenir à partir d'un individu représenté par un point intérieur de $c_{i}$ un individu de $c_{k}$, d'où il existe un chemin issu de $c_{k / 1}$ qui permet d'atteindre $c_{i / l} \cdot c_{k / 1}$ est donc une racine de $G^{(k)}$.

Remarque : Bien que $G^{(k)}$ admette des cycles (Berge, $1983 ; \mathrm{p} 8$ ), il n'admet aucun circuit (Berge, $1983 ; \mathrm{p} 8)$, ce qui permet de démontrer que $c_{k / 1}$ est l'unique racine de $G^{(k)}$.

\section{Proposition 2}

Si le sous-graphe $G_{l}^{(k)}$ n'est pas réduit au graphe sans arc, il existe au moins un chemin qui part de $c_{k / 1}$ pour atteindre chacun des sommets de $S_{l}^{(k)}$.

\section{Démonstration}

Puisque $G^{(k)}$ a une racine, il est quasi-fortement connexe (Berge, 1983; p 30). On a donc :

$$
\begin{gathered}
\forall c_{i / p} \in E^{(k)} ; c_{i / p} \neq c_{k / 1} \\
d_{G^{(k)}}^{-}\left(c_{i / p}\right) \geq 1
\end{gathered}
$$

où $d_{H}^{-}(x)$ est le demi-degré intérieur du sommet $x$ dans le graphe $H$.

Comme nous ne nous intéressons qu'aux sous-graphes $G_{l}^{(k)}$ ayant au moins $c_{k / 1}$ comme sommet et dont les sommets ont même demi-degré intérieur que dans $G^{(k)}$, on en déduit que $c_{k / 1}$ est aussi la racine de $G_{l}^{(k)}$ (si ce dernier n'est pas le graphe sans arc), ce qui est suffisant pour conclure.

Nous proposons de faire une «lecture» de chaque sous-graphe $G_{l}^{(k)}$ en lui associant un effet du modèle, noté de la manière suivante : 
- le sous-graphe sans arc est associé à l'effet $I_{c_{k}}^{c_{k}}$ qui d'un point de vue statistique représentera l'effet de variabilité intra;

- pour les autres sous-graphes, l'effet est une interaction dont l'ordre est égal au nombre de sommets de $S_{l}^{(k)}$ moins 1 . Chaque sommet de $S_{l}^{(k)}$ est associé à un facteur de cette interaction que l'on indice supérieurement par $c_{k}$ et inférieurement par le cercle du sommet considéré. Le facteur est noté par réunion des marques trouvées dans la table $T^{(k)}$ pour un chemin reliant $c_{k / 1}$ et le sommet considéré et l'interaction est notée comme la suite des facteurs séparés par des points.

Remarque : La notation choisie pour les interactions et les facteurs de ces interactions est purement conventionnelle. Ce qu'il faut retenir, c'est l'importance pour un effet du modèle de l'ensemble $S_{l}^{(k)}$ associé au sous-graphe $G_{l}^{(k)}$, puisqu'il détermine l'ordre de l'effet et les types de géniteurs représentant les individus qui créent les niveaux de cet effet.

\section{Proposition 3}

Il existe une bijection entre un sous-graphe $G_{l}^{(k)}$ et son ensemble $S_{l}^{(k)}$.

\section{Démonstration}

Par définition, à chaque $G_{l}^{(k)}$ est associé un $S_{l}^{(k)}$ et un seul.

Supposons que la réciproque soit fausse, alors il existe $S_{l}^{(k)}$ ensemble des sommets de demi-degré extérieur égal à 0 pour 2 sous-graphes différents $G_{l}^{(k)}$ et $G_{l^{\prime}}^{(k)}$. Il existe donc un sommet $c_{0}$ qui appartient par exemple, à $E_{l}^{(k)}$ mais n'appartient pas à $E_{l^{\prime}}^{(k)}$. La seule solution pour que de ce sommet, il n'existe aucun chemin vers un sommet de $E_{l^{\prime}}^{(k)}$ (ce qui modifierait son demi-degré intérieur) est que $c_{0}$ appartienne à un circuit qui ne passe par aucun des sommets de $E_{l^{\prime}}^{(k)}$. D'où la contradiction, car la relation «être descendant par une manipulation élémentaire» est anti-symétrique, il ne peut donc exister de circuit dans les graphes lui étant associés.

On trouvera dans la figure 4 (resp 5), les sous-graphes et les effets du modèle pour la valeur génétique des individus du cercle $c_{6}\left(\operatorname{resp} c_{7}\right)$ de la figure 3 .

Intéressons-nous par exemple à un individu de $c_{7}$, on peut lire le modèle trouvé en disant que sa valeur génétique est égale à la somme :

- de l'effet de son ancêtre dans $c_{1}$ utilisé en tant que l'ancêtre d'autofécondation de son père (ou de sa mère selon le chemin choisi);

- de l'effet de l'ancêtre dans $c_{4}$ utilisé en tant que père;

- de l'effet de l'ancêtre dans $c_{4}$ utilisé en tant que mère;

- de l'effet de l'interaction entre ses 2 ancêtres dans $c_{4}$ dont l'un est son père et l'autre sa mère;

- et de son effet individuel dans $c_{7}$.

Où encore, si l'on note $i$ un individu de $c_{1}, i j$ un individu de $c_{4}, i j j^{\prime} k$ un individu de $c_{7}$ si $i j$ est son père et $i j^{\prime}$ sa mère, la valeur génétique d'un individu de $c_{7}$ est égale à :

$$
A P_{i}^{c_{7}}+P_{i j}^{c_{7}}+M_{i j^{\prime}}^{c_{7}}+P_{i j}^{c_{7}} \cdot M_{i j^{\prime}}^{c_{7}}+I_{i j j^{\prime} k}^{c_{7}}
$$


Ce qui est le modèle statistique complet naturel pour les individus issus d'un croisement dans une famille obtenue par autofécondation.

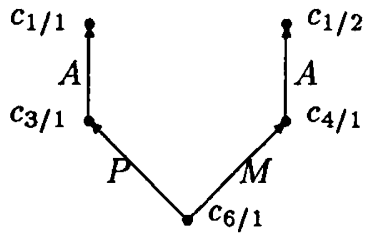

$A P_{c_{1}}^{c_{6}} \cdot A M_{c_{1}}^{c_{6}}$

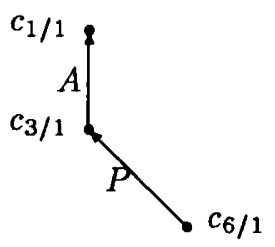

$A P_{c_{1}}^{c_{6}}$

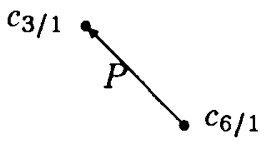

$P_{c_{3}}^{c_{6}}$

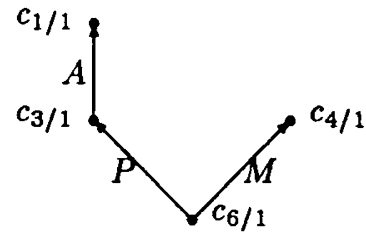

$A P_{c_{1}}^{c_{6}} . M_{c_{4}}^{c_{6}}$

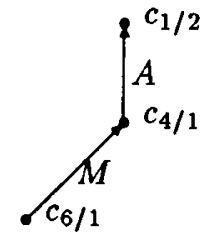

$A M_{c_{1}}^{c_{6}}$

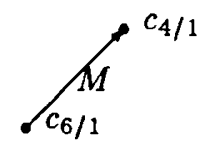

$M_{c_{4}}^{c_{6}}$

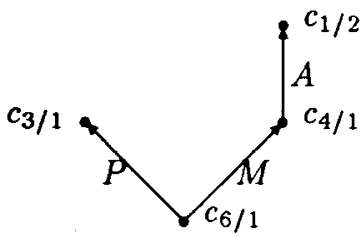

$P_{c_{3}}^{c_{6}} \cdot A M_{c_{1}}^{c_{6}}$

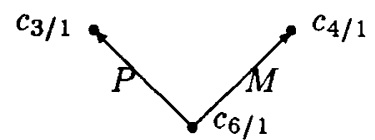

$P_{c_{3}}^{c_{6}} \cdot M_{c_{4}}^{c_{6}}$

- $c_{6 / 1}$

$I_{c_{6}}^{c_{6}}$

Fig 4. Sous-graphes et effets du modèle pour la valeur génétique des individus de $c_{6}$.

\section{Trouver les covariances entre valeurs génétiques}

La méthode des sous-graphes permet d'écrire un modèle et le caractère aléatoire des effets trouvés est une hypothèse classique des modèles de génétique quantitative. Cependant, il reste à déterminer pour la valeur génétique de chacune des plantes modélisées, la corrélation avec la valeur génétique des autres plantes.

Nous allons énoncer 3 règles algébriques qui permettent de construire facilement la matrice de variance-covariance entre valeurs génétiques, lorsque le modèle a été trouvé en utilisant la méthode des sous-graphes. L'objet de la deuxième partie de l'article de Goffinet et Mangin, 199x (document interne), est de transformer ces 


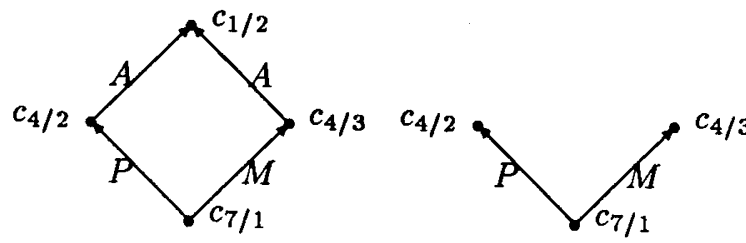

$$
A P_{c_{1}}^{c_{7}} \quad P_{c_{4}}^{c_{7}} \cdot M_{c_{4}}^{c_{7}}
$$

ou

$$
A M_{c_{1}}^{c_{7}}
$$

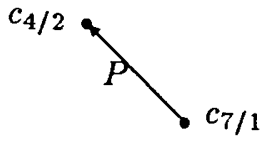

$P_{c_{4}}^{c_{7}}$

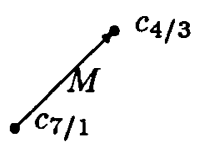

- $c_{7 / 1}$

$M_{c_{4}}^{c_{7}}$

$I_{c_{7}}^{c_{7}}$

Fig 5. Sous-graphes et effets du modèle pour la valeur génétique des individus de $c_{7}$.

3 règles en 3 propositions, dont les démonstrations respectives sont basées sur le tirage aléatoire dans des populations infinies.

Pour ne pas alourdir la suite de l'article, nous allons définir des notations simplifiées pour les effets des modèles obtenus pour chacun des cercles du schéma. Notation : Un effet obtenu par la méthode des sous-graphes est entièrement défini par :

- des facteurs notés de façon simplifiée $F^{1}, F^{2}, \ldots, F^{n}$, qui correspondent à la réunion, indicée supérieurement par $c^{k}$, des marques de la table $T^{(k)}$ pour un chemin choisi entre $c_{k / 1}$ et chacun des sommets de $S_{l}^{(k)}$;

- des indices inférieurs $c_{1}, c_{2}, \ldots, c_{n}$ attachés à chacun des facteurs, qui correspondent aux cercles dans lesquels sont pris les sommets de $S_{l}^{(k)}$.

L'effet est alors noté : $F_{c_{1}}^{1} \cdot F_{c_{2}}^{2} \ldots F_{c_{n}}^{n}$

\section{Covariance intra}

Considérons : le $n$-uplet $E=\left(c_{1}, \ldots, c_{n}\right)$, le $n$-uplet $F=\left(F^{1}, \ldots, F^{n}\right)$, et l'ensemble des permutations possibles d'un ensemble de $n$ éléments dans lui-même. Notons ces permutations $\sigma_{0}=I d, \sigma_{1}, \ldots, \sigma_{n !-1}$. 
Règle 1

Il y a une covariance intra associée à la permutation $\sigma_{i}$ ou variance pour $i=0$, entre deux niveaux de l'effet tels que :

$$
\left\{\begin{array}{ccc}
\text { niveau de } F_{1} & = & \text { niveau de } F^{\sigma_{i}(1)} \\
\vdots & = & \vdots \\
\text { niveau de } F^{n} & = & \text { niveau de } F^{\sigma_{i}(n)}
\end{array}\right.
$$

Remarquons cependant que, si $\sigma_{i}$ n'est pas sa propre inverse, (1) permet d'obtenir que :

$$
\left\{\begin{array}{ccc}
\text { niveau de } F_{1} & = & \text { niveau de } F^{\sigma_{j}(1)} \\
\vdots & & \vdots \\
\text { niveau de } F^{n} & = & \text { niveau de } F^{\sigma_{j}(n)}
\end{array}\right.
$$

où $\sigma_{j}=\sigma_{i}^{-1}$. Chaque covariance intra peut donc être associée à une permutation involutive ou 2 permutations inverses.

Prenons, par exemple, une interaction d'ordre 3, $P P_{c_{1}} \cdot M P_{c_{1}} \cdot P M_{c_{1}} \cdot M M_{c_{2}}$, effet présent dans le modèle pour la valeur génétique des descendants obtenus par pollinisation contrôlée entre un père pris dans les descendances d'un croisement à l'intérieur d'une population $A$ (représentée par le cercle $c_{1}$ ) et une mère prise dans les descendances d'une pollinisation contrôlée entre un père pris dans $A$ et une mère prise dans une population $B$ (représentée par $c_{2}$ ). La règle énoncée nous dit que pour la covariance de la valeur génétique de 2 plantes soit en partie due à l'interaction d'ordre 3 considérée, il faut et il suffit qu'elles aient les mêmes grandsparents. Par contre, la covariance ne sera pas identique entre la valeur génétique de 2 plantes qui ont les mêmes grands-parents maternels mais dont les grands-parents paternels sont échangés, et celle de 2 plantes qui ont les mêmes grands-mères mais dont les grands-pères sont échangés.

Définissons la relation d'équivalence $c_{i} \cong c_{j}$ par :

- soit $c_{i}=c_{j}$,

- soit $c_{i}$ est équivalent $c_{j}$, c'est-à-dire qu'ils représentent les mêmes individus.

Considérons :

$E$ un $n$-uplet $\left(c_{1}, \ldots, c_{n}\right)$ et $E^{\prime}$ un $n$-uplet $\left(c_{1}^{\prime}, \ldots, c_{n}^{\prime}\right)$, et définissons $E \cong E^{\prime}$ par :

$$
\forall_{i}, c_{i} \cong c_{i}^{\prime}
$$

\section{Proposition 4}

Il n'est pas nécessaire de passer en revue toutes les $n$ ! permutations car seuls les permutations telles que $\sigma_{i}(E) \cong E$ sont associées à une covariance intra ou à la variance. 


\section{Démonstration}

Par définition, le niveau d'un facteur est associé à un des individus représentés par un point intérieur au cercle qui indice inférieurement le facteur.

Pour que tout $l \in[1, \ldots, n]$ :

niveau de $F^{l}=$ niveau de $F^{\sigma_{i}(l)}$ il faut que :

$c_{l}$ et $c_{\sigma_{i}(l)}$ représentent les mêmes individus. Ce qui peut se traduire par $c_{l} \cong c_{\sigma_{i}(l)}$. Cette relation d'équivalence étant nécessaire pour tout $l$, on obtient : $E \cong \sigma_{i}(E)$.

\section{Covariance inter}

\section{Règle 2}

Seuls les effets de même ordre covarient entre eux.

Considérons : le $n$-uplet $E=\left(c_{1}, \ldots, c_{n}\right)$; le $n$-uplet $E^{\prime}=\left(c_{1}^{\prime}, \ldots, c_{n}^{\prime}\right)$; le $n$-uplet $F=\left(F^{1}, \ldots, F^{n}\right)$; le $n$-uplet $F^{\prime}=\left(F^{\prime 1}, \ldots, F^{\prime n}\right)$; et l'ensemble des permutations possibles d'un ensemble de $n$ éléments dans lui-même.

\section{Règle 3}

Il y a une covariance inter entre les niveaux des effets tels que :

$$
\left\{\begin{array}{ccc}
\text { niveau de } F_{1} & = & \text { niveau de } F^{\prime \sigma_{i}(1)} \\
\vdots & = & \vdots \\
\text { niveau de } F^{n} & = & \text { niveau de } F^{\prime \sigma_{i}(n)}
\end{array}\right.
$$

(3) n'est plus symétrique entre $F$ et $F^{\prime}$; chaque covariance inter est donc associée à une et une seule permutation.

\section{Proposition 5}

De même que pour les covariances intra, seules et permutations telles que $\sigma_{i}(E) \cong$ $E^{\prime}$ sont associées à une covariance inter.

\section{Conclusion}

Le modèle statistique trouvé à l'aide de cette méthode peut comporter un grand nombre de paramètres de variance-covariance, mais il est très simple de prendre en compte toutes les considérations ou hypothèses génétiques qui s'expriment comme l'égalité de 2 de ces paramètres ou comme la nullité d'un des paramètres.

Cette modélisation a été présentée dans un cadre univariable, mais la généralisation à plusieurs variables est immédiate. En effet, lorsque plusieurs variables sont modélisées simultanément, les effets trouvés par la méthode des sous-graphes doivent être regardés comme des vecteurs d'effets dont chaque élément est associé à une des variables. Quant aux règles pour trouver les variances-covariances entre 
apparentés, elles restent identiques, si ce n'est le fait qu'une permutation est alors associée à une matrice de variance-covariance.

Notons, pour finir, que la méthode que nous avons exposée existe sous la forme d'un module d'un logiciel (Select, 1991) qui génère automatiquement le modèle statistique après entrée du protocole à l'aide d'une interface graphique.

\section{REMERCIEMENTS}

Nous remercions l'un des lecteurs pour ses remarques constructives et pertinentes, ainsi que M Schnuremberger pour la production des figures.

\section{RÉFÉRENCES}

Berge C (1983) Graphes. Gauthier-Villars, Paris, 399 p

Cannings C, Thompson EA, Skolnick MH (1978) Probability functions on complex pedigrees. Adv Appl Prob 10, 26-61

Gallais A (1977) Amélioration de l'efficacité des schémas de sélection récurrentes. Ann Amélior Plant 27, 477-481

Gallais A (1990) Théorie de la sélection en amélioration des plantes. Masson, Paris, $588 \mathrm{p}$

Henderson CR (1973) Sire evaluation and genetics trend. Proc Anim Breed Genet Symp in honor of JL Lush. Am Soc Anim Sci and Am Dairy Sci Assoc, Champaign, IL

Maruyama T, Yasuda N (1970) Use of graph theory in computation of inbreeding and kinship coefficients. Biometrics 26, 209-220

Select group (1991) Notice d'utilisation du logiciel Select. INRA Biométrie, Toulouse, France 
Book review

RK Selander, AG Clarke and TS Whittam (eds) (1991) Evolution at the Molecular Level. Sinauer Associates Inc, Sunderland, Massachussetts, xii + 350 p.

Over a period of a few decades, molecular analyses have dramatically changed the study of evolutionary biology, and as sequencing techniques become easier and cheaper to employ and as the opportunity to use mummified and fossilized material becomes available, much more can be expected. Although there has been an explosion of data, theory and data analysis techniques have progressed such that we have new understandings, not just new facts. This volume, comprising 13 reviews by specialists in their area, demonstrates the pervasiveness and influence of molecular methods. It had its origins in a symposium held in 1989, but is well up to date in this rapidly changing field.

About half the book is devoted to the evolution and population genetics of the non-eukaryotic genome, including the HIV retrovirus (Yokoyama), bacteria (Woese, Selander, Hartl, Milkman and colleagues), and the mitochondrial and chloroplast organelles (Birkby, Clegg and colleagues). The Drosophila transposable elements (Charlesworth and Langley) could be regarded as spanning the bridge to the eukaryotic genome. Without molecular methods none of these topics could be considered sensibly at any level, for example an evolutionary classification of bacteria was not possible using chemical, morphometric or immunological methods. Now evolution of ribosomal RNA has been used to construct basic trees for archaebacteria and bacteria and, at a finer level, trees for Salmonella species. These often show no close phylogenetic relationship among individual human adapted serovars, implying independent evolution or horizontal transfer.

The topics reviewed on the eukaryotic genome include a discussion of the globin families (Hardison) which have been so fruitful in showing, for example, the evolution of duplicated genes and of pseudogenes. The MHC system raises problems for population geneticists because of its diversity (Nei, Hedrich and colleagues). Meiotic drive, in contrast, has been a phenomenon looking for a molecular explanation, but progress is being made (Wu and Kennet). Kreitman shows how information at the molecular level can be used by population geneticists to distinguish between (resolve) neutral and selected genes. I still get the nagging feeling that the collection of ever more data will not lead to resolution of the neutralist/selectionist controversy, for there is no single answer, but he is more optimistic.

Overall, I found this an excellent book, likely to be of use to both professionals and students.

William G Hill, University of Edinburgh 
(C) Elsevier/INRA

Calendar

XLIII ${ }^{e}$ réunion annuelle de la Fédération européenne de zootechnie (FEZ)

(13-17 septembre 1992, Madrid, Espagne)

Programme des séances de la Commission de génétique animale de la FEZ :

- séance I : (Conjointement avec la Commission de production Porcine). Estimation de la valeur génétique des porcs;

- séance II : Progrès en génétique moléculaire;

- séance III : Systèmes d'élevage et de production pour les bovins des régions tropicales et subtropicales;

- séance IV : Génétique des caractères de reproduction;

- séance V : Communications libres;

- séance VI : «Boîte à idées» et séance administrative.

\section{XLIIIrd EAAP Annual Meeting}

(13-17 September 1992, Madrid, Spain)

\section{Programme of the Commission on Animal Genetics :}

- session I : (Jointly with the Commission on Pig Production). Estimation of breeding values in pigs;

- session II : Advances in molecular genetics;

- session III : Breeding and production systems for cattle in the tropics and subtropics;

- session IV : Genetics of reproductive traits;

- session V : Free communications;

- session VI : "Ideas box" and Business meeting. 
The journal Genetics Selection Evolution publishes original articles, review articles, short notes, and letters to the editor in English and in French.

Address manuscripts to :

L Ollivier, INRA, Station de Génétique Quantitative et Appliquée, 78352 Jouy-en-Josas Cedex, France.

\section{GENERAL PRESENTATION}

Three complete sets of the manuscript must be submitted. The manuscript must be typeset double-spaced, with margins of at least $3.5 \mathrm{~cm}$ at top, bottom and sides for editor's markings.

The layout should be arranged as follows : title page, summary in the language of the article, title and summa$y$ in the other language (in French if the article is written in English and vice versa), introduction, materials and methods, results, discussion, acknowledgments, references, figure legends, tables, figures.

$N B$ : Please note the absence of full stops after all abbreviations.

\section{ritle page}

The title page should include the following: title, authors' names, institution and department where the study was carried out, a short title for runaing headlines.

\section{Summaries}

The summaries (not more than 200 words in the language of the article and not exceeding 500 words in the ther language) should be in a suiable form for abstracting services. Please avoid paragraphs and footnotes. References and undefined bbreviations are not allowed.

\section{Key words}

Up to 5 key words should be supolied, to assist the reader and faciliate information retrieval. Key words nay be taken from the title, summary or text. Please avoid the plural form.

\section{References}

References in the text should include he author(s) name(s) and year of ublication. When more than 2 authors are cited, the first author's name should appear followed by " $e t$ $a l$ ". A number of references cited together should be chronologically set out. Ex: Hartl et al (1986); Hartl and Reimoser (1988); Hartl (1990a).

In the bibliography, author's names are listed in alphabetical order, as follows: publications of a single author in chronological order, publications of the same author with one co-author in chronological order, publications of the author with more than one coauthor in chronological order. All entries in the reference list must correspond to references in the text and vice versa.

The spelling of authors' names and dates must be the same both in the text and in the reference list.

The titles of journals should be abbreviated according to the rules of Biosciences Information Service (Biosis). Words for which no abbreviation is given should be written in full.

Examples are given below of the layout and punctuation to be used in the references:

- article (please mention all the authors)

Lefort G, Ollivier L, Sellier P (1975) Analyse du comportement et de la frequence des gènes à effets visibles dans les fratries de germains et de demi-germains. Ann Génét Sél Anim 7, 365-377

- book

Charlesworth B (1980) Evolution in Age-Structured Populations. Cambridge Univ Press, Cambridge

\section{- chapter in a book}

Lemeunier F, David JR, Tsacas L, Ashburner M (1986). The melanogaster species subgroup. In: The Genetics and Biology of Drosophila (Ashbumer M, Thompson JR, Carson HL, eds) Academic Press, London, vol 3, 147-256

\section{- symposium or congress paper}

Ollivier L, Bolet G (1981) La sélection sur la prolificité chez le porc: résultats d'une expérience de sélection sur dix générations. In: 13 Journées de la Recherche Porcine en France. Paris, 4-5 February 1981. Institut Technique du Porc, Paris, 261-267

\section{Illustrations (figures and tables)}

Illustrations (1 original and 2 good copies), should be numbered in Arabic numerals for tables. They should be referred to in the text by their number and their ideal position indicated in the margin. Lettering (symbols, numbers, etc) should not differ from figure to figure and should be of sufficient height and thickness to remain legible after reduction. Figures should be presented in the form of drawings on drawing or tracing paper or as sharp prints.

Photographs and half-tones should contain good contrast; line drawings should have a white background. Tables should not exceed 84 characters per line. Figure legends should appear on the same page and should be explicit so that the illustrations are comprehensible without reference to the text. Authors will be charged for colour prints. Figures and tables published elsewhere cannot be accepted without prior consent of the publisher and the author(s).

The editorial board maintains the option of returning, before evaluation, manuscripts to authors who do not comply with these recommendations. The author is advised to keep one manuscript and a set of figures.

\section{PROOFS AND REPRINTS}

Proofs will be sent to the author indicated on the title page. They should be carefully corrected and returned to the publisher within 48 hours of reception. If this period is exceeded, the galleys will be printed without the author's corrections. Should substantial changes in the original manuscript be requested, they will be made at the author's expense. Fifty reprints per contribution are available free of charge. An order form for additional reprints will accompany the proofs.

There is no page charge for text and black-and-white figures.

\section{COPYRIGHT}

As soon as the article is published, the author is considered to have transferred his rights to the publisher. Requests for reproduction should be sent to the latter. 
La revue Genetics Selection Evolution publie des articles de recherche, des articles de synthèse, des courtes notes, des tribunes libres, et des lettres à la Rédaction en anglais et en français.

Adresse pour la soumission des manuscrits :

L Ollivier, INRA, station de génétique quantitative et appliquée, 78352 Jouyen-Josas Cedex, France

\section{PRÉSENTATION GÉNÉRALE}

Le manuscrit est soumis en 3 exemplaires. Il est dactylographié en double interligne avec des marges d'au moins $3,5 \mathrm{~cm}$ en haut, en bas et sur les côtés pour les annotations de la rédaction.

La présentation matérielle du manuscrit est la suivante : page de titre, résumé dans la langue de l'article, titre et résumé dans l'autre langue que celle de l'article (en anglais si l'article est rédigé en français et inversement), introduction, matériel et méthodes, résultats, discussion, remerciements, références, légendes des figures, figures, tableaux.

\section{NB: On notera l'absence de points abréviatifs.}

\section{Page de titre}

La page 1 du manuscrit indique le titre, le nom des auteurs, l'adresse de l'établissement ou a été effectué le travail, un titre abrégé (titre courant).

\section{Résumés}

Les résumés (moins de 200 mots dans la langue de l'article et moins de 500 mots dans l'autre langue) doivent être présentés sous une forme adaptée à une exploitation par des services documentaires. Eviter les paragraphes, les renvois en bas de page. Les références bibliographiques et les abréviations non définies ne sont pas admises.

\section{Mots clés}

Cinq mots clés au plus doivent être fournis, afin de faciliter l'indexation de l'article dans les fichiers documentaires. Les mots clés peuvent provenir du titre, du résumé ou du texte. Eviter le pluriel.

\section{Références (bibliographiques)}

Dans le texte, les références sont citées par les noms d'auteurs suivis de l'année de publication. La citation des articles écrits par plus de 2 auteurs est faite par le nom du premier auteur suivi de «et al.». Les références citées ensemble dans le texte doivent être ordonnées chronologiquement. Ex. : Hartl et al (1986); Hartl et Reimoser (1988); Hartl (1990a).

La liste des références est organisée par ordre alphabétique des noms d'auteurs et par ordre chronologique pour un auteur donné. Si un auteur a écrit certains de ses articles avec un ou plusieurs coauteurs, l'ordre est le suivant : articles de l'auteur seul, classés par ordre chronologique; articles du même auteur avec un coauteur, classés par ordre chronologique; articles de l'auteur avec plus d'un coauteur, classés par ordre chronologique.

Toutes les reférences de la liste doivent correspondre à des références citées dans le texte et vice versa.

Les titres des périodiques doivent être abrégés selon les normes de Biosciences Information Service (Biosis). Ecrire en toutes lettres les mots pour lesquels aucune abréviation n'est donnée.

Le style et la ponctuation des références sont conformes aux modèles illustrés dans les exemples suivants :

- article (indiquer tous les auteurs)

Lefort G, Ollivier L, Sellier P (1975) Analyse du comportement et de la fréquence des gènes à effets visibles dans les fratries de germains et de demi-germains. Ann Génét Sél Anim 7, 365 377

\section{- ouvrage}

Charlesworth B (1980) Evolution in age-structured populations. Cambridge Univ Press, Cambridge

\section{- chapitre d'ouvrage}

Lemeunier F, David JR, Tsacas L, Ashburner M (1986) The melanogaster species subgroup. In : The genetics and biology of Drosophila (Ashburner M, Thompson JR, Carson HL eds) Acad Press, Londres, Vol 3, 147256

- communication (publiée) d'un colloque ou congrès

Ollivier L, Bolet G (1981) La sélection sur la prolificité chez le porc: résultats d'une expérience de sélection sur dix générations. In : 13e Journées de la recherche porcine en France. Paris, 45 février 1981, Institut technique du porc, Paris, 261-267

\section{Illustrations (figures et tableaux)}

Les illustrations ( 1 original et 2 bonnes copies) seront numérotées en chiffres arabes pour les figures, romains pour les tableaux, et indexées dans le texte par rappel de leur numéro. Indiquer dans la marge du manuscrit leur emplacement idéal. Le lettrage (symboles, chiffres, etc.) doit être uniforme pour toutes les figures et de taille suffisante pour rester lisible après réduction. Les figüres seront présentées sous forme de dessins, tracés ou photographies. Les photos en demi-teinte devront être suffisamment contrastées; les photos de dessins ou tracés auront un fond blanc. Les tableaux ne doivent pas comporter plus de 84 caractères par ligne. Les légendes doivent être claires, permettant aux illustrations d'être compréhensibles par elles-mêmes; elles devront toutes être groupées sur une même feuille. Les figures en couleurs seront à la charge de l'auteur. Aucune reproduction d'une figure ou d'un tableau déjà publié ne peut être acceptée sans l'autorisation écrite de l'éditeur et des auteurs.

Le comité de rédaction se réserve le droit de renvoyer aux auteurs, avant toute lecture, les manuscrits qui ne seraient pas conformes à ces recommandations. Il demande que les auteurs conservent un exemplaire du manuscrit et des illustrations.

\section{ÉPREUVES D'IMPRIMERIE ET TIRÉS À PART}

Les épreuves d'imprimerie sont envoyées à l'auteur indiqué sur le manuscrit. Elles doivent être soigneusement corrigées et renvoyées à l'éditeur dans les 48 heures qui suivent la réception. En cas de retard, l'éditeur se réserve, après sa propre correction, de procéder à l'impression sans les corrections d'auteurs. Si l'auteur requiert des modifications importantes, il en supportera la charge financière. Les 50 premiers tirés à part sont grátuits. Un bon de commande pour des exemplaires supplémentaires est joint aux épreuves.

La publication des textes et des figures en noir et blanc est gratuite.

\section{DROITS DE REPRODUCTION}

Dès que l'article est publié, l'auteur est réputé avoir cédé ses droits à l'éditeur. Les demandes de reproduction seront adressées à celui-ci. 


\section{Sommaire}

Propagation de facteurs sexuels parasites dans les populations d'Armadillidium vulgare Latr (Crustacé, Oniscoïde) : conséquences sur le taux de masculinité

T Rigaud, JP Mocquard, P Juchault (Poitiers, France) ................

Estimation de l'héritabilité dans la population initiale en utilisant seulement les données des générations subséquentes

L Gomez-Raya, LR Schaeffer, EB Burnside (Guelph, Canada)...........

Aspects théoriques de l'application du test de liaison génétique par les couples de germains aux espèces animales domestiques

$K U$ Götz, L Ollivier (Göttingen, Allemagne; Jouy-en-Josas, France).......

Sélection massale divergente pour la conformation chez la carpe commune

Y Ankorion, R Moav, GW Wohlfarth (Hof Hacarmel, Israël)...........

Paramètres génétiques des caractères de croissance, de gavage et de foie gras dans le croisement de deux souches d'oies (Anser anser) sélectionnées (en français)

$R$ Rouvier, B Poujardieu, D Rousselot-Pailley, P Larrue, D Esteve (Castanet-

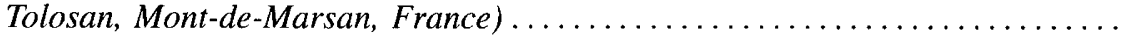

Schémas de sélection : de la représentation généalogique au modèle statistique. Elaboration du modèle (en français)

B Mangin, P Vincourt (Castanet-Tolosan, Blagnac, France)........... 71

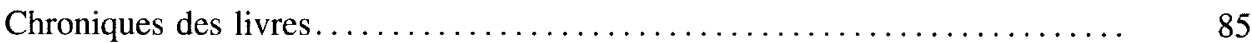

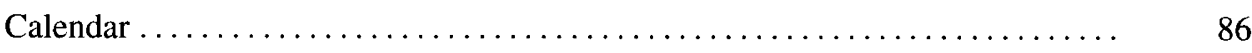




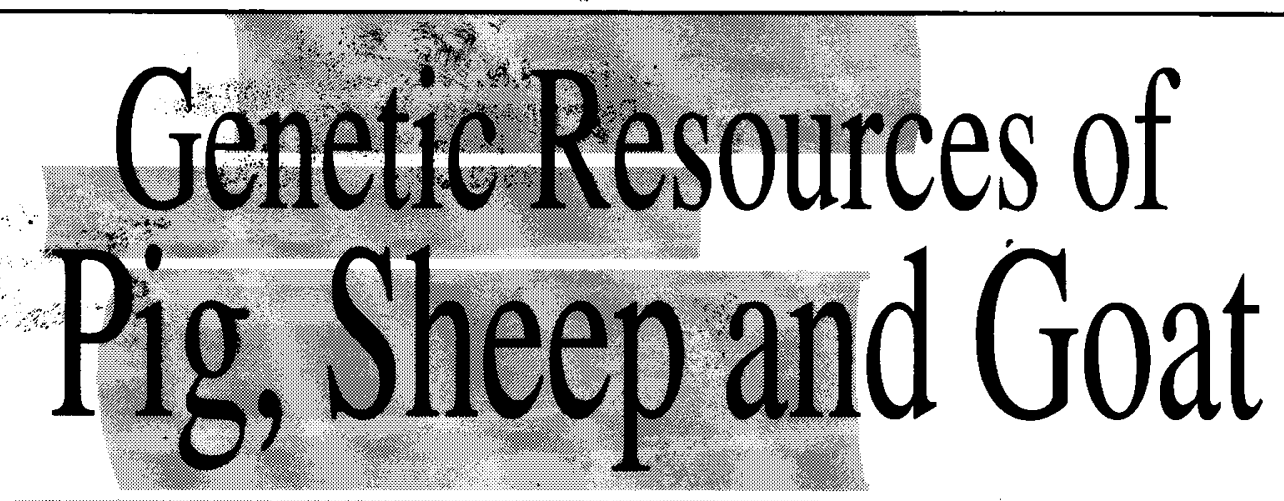

edited by K. Maijala, Academy of Finland, Helsinki, Finland

WORLD ANIMAL SCIENCE Vol. B8

\author{
1991 xviii + 556 pages \\ Price: US $\$ 210.00 /$ Dfl. 410.00 \\ ISBN 0-444-88279-0
}

The book concerns genetic resources in three different species of farm animals. In each species, civilization and domestication, breed classification and distribution, breed differences in adaptation and productivity, breed evaluation and utilzation, new breed development, breed identification, breeding programmes, gene identification, international exchange of breeding stacks, and conservation of genetic resources are discussed. In addition, the social aspeets sheepbreeding are discussed, and in sheep and goats the evolution of fibres and skins as well as milk production.

Contents: Ceneral Preface: Preface to Volume B8. 1. Evolution and Domestication, and Introduction (P. Jonsson). 2. Civilization and Domestication of Pigs (P. Jonsson). 3. Pig Breeds of the World, Their Distribution and Adaptation (J.W.B. King). 4. Evaluation and Utilization of Pig Breeds (P. Glodek). 5. New Breed Development in Pigs (W.E. Rempel and K. Maijala). 6. Gene Identification in Pigs (R. Hanset). 7. Breed Identification and Development in Pigs (P. Sellies and M.F. Bothschild). 8. Conservation of Genetic Resources in Pigs and Sheep (D.E. Steane). 9. Domestication, History and Breed Evolution in Sheep (M.I. Ryder). 10. Classification and Distribution of Sheep Breeds (I.L. Mason). 11. Breed Differences in Adaptation of Sheep (C.E. Terrill and J. Slee). 12. Breed Differences in Prolificacy of Sheep (I.L. Mason). 13. Milk Breeds of Sheep (J.G. Boyazoglu). 14. Comparisons for Meat Production in Sheep (C.E. Terrill and K. Maijala). 15. Genetic Resources for Wool Production (C.H.S. Donling and B.C. Jefferies). 16. Fur Breeds of Sheep (J.A. Nel and O. Danell). 17. Breed Structure, Dynamics and New Breed Development in Sheep (K. Maijala and C.E. Terrill). 18. Milk Recording, Breeding and Selection Schemes for Milk Breeds (J.G. Boyazoglu). 19. Breeding Programmes in Nordic Countries Based on Field Production Recording (O. Danell). 20. Stratified Breed Utilization Schemes in Great Britain (D. Croston and T.T. Treacher). 21. State Farm Breeding in Eastern European Countries (S. Kukovies). 22. International Exchange of Genetic Materials (J.C. Flamant). 23. Gene Identification in Sheep (S. Adalsteinsson). 24. Considering the Social Aspects of Sheep Breeding Programmes (J.C. Mlamant). 25. Domestication and History of Goats (K. Nozawa). 26. Classification and Distribution of Goat Breeds (I.1. Mason). 27. Breed Differences in Adaptation of Goats (C.F. Gall). 28. Breed Differences

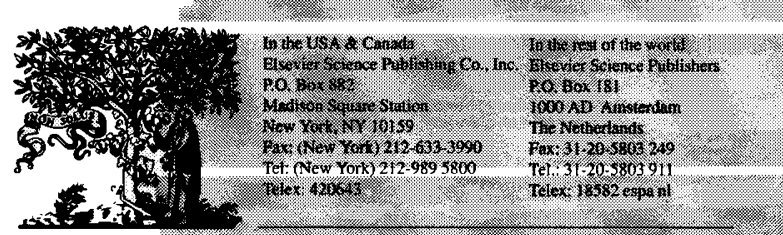

ELSEVIER SCIENCE PUBLISHERS in Productivity in Goats (C. Denvendra). 29. Evolution of Mohair, Cashmere and Skins (S.I. Arbiza). 30. Evaluation and Utilization of Goat Breeds (A.R. Quartermain). 31. Gene Identification of Goats (G. Ricordeau). 32. Breeding Programmes and Production Recording in Goats (G. Ricordeau). 33. Maintenance of Cenetic Resources in Goats (A.R. Quatermain). List of Contributors. Subject Index.

The Dutch guilder price is definitive. UST prices are subject to exchange rate fluctuations. Prices are excl. B.T.W. for Dutch customers 